\title{
Cognitive assessment in asymptomatic COVID-19 subjects
}

\author{
Sridhar Amalakanti ${ }^{1}$ (D) $\cdot$ Kesava Venkata Raman Arepalli $^{2} \cdot$ Jyothi Priya Jillella $^{3}$
}

Received: 4 September 2020/Accepted: 26 January 2021/Published online: 15 February 2021

(C) Indian Virological Society 2021

\begin{abstract}
Neurological features of COVID-19 have been reported in addition to the respiratory manifestations, but cognitive dysfunction has been scarcely described. And cognitive assessment has not been studied in asymptomatic subjects. We compared the cognitive assessment scores between asymptomatic SARS-CoV-2 infected subjects with that of controls to detect mild cognitive impairment by the Montreal Cognitive Assessment test. Asymptomatic COVID-19 subjects secured lower scores in certain domains of the MoCA in comparison with the controls. The domains were visuoperception $(2.4 \pm 0.7$ vs $2.8 \pm 0.7$, $p=0.032)$, naming $(3.6 \pm 0.5$ vs3.9 $\pm 0.2, p=0.016)$ and fluency $(0.9 \pm 0.6$ vs1.6 $\pm 0.7, p=<0.001)$. Also, older aged COVID-19 positive subjects scored lower in the MoCA when compared to the younger people. Our study shows that even otherwise asymptomatic COVID-19 subjects have cognitive deficits in certain subdomains and suggests the need for a detailed psychometric assessment especially in the elderly population.
\end{abstract}

Keywords COVID-19 brain · COVID-19 neurological . COVID-19 psychiatric · COVID-19 neuropsychiatric . COVID-19 cognition

Supplementary Information The online version of this article (https://doi.org/10.1007/s13337-021-00663-w).

Sridhar Amalakanti

iamimenotu@gmail.com

1 Institute of Bioinformatics, Discoverer Building, 7th Floor, International Tech Park, Whitefield, 560066 Bengaluru, India

2 Department of General Medicine, Great Eastern Medical School and Hospital, Ragolu, India

3 GNM, Harika College of Physiotherapy, Guntur, India
COVID-19, caused by SARS-CoV-2 has killed millions of people across the world in less than a year. It has affected almost all of the human population directly or indirectly. Directly by causing a spectrum of disorders ranging from no symptoms to death. Indirectly by resulting in sickness or loss of a family member or socioeconomic turbulence due to restricted movement of parcels and people [1]. The people afflicted with SARS-CoV-2 were reported to have respiratory symptoms in the earliest studies. Thereafter a number of extra respiratory features were also observed. These included neurological deficits also. Some of these were stroke, seizures, neuropathy and movement disorders [2]. Recent pathological studies have shown the neurotropism of SARS-CoV-2, thus establishing a direct pathophysiological basis to the neurological features [3].

Neurological features of higher mental functions were reported by Zhou et al. who performed a study on the cognitive functions of 29 COVID-19 recovered patients. The patients secured lower scores in Continuous Performance Test (which is used to assess attention and impulse) than the controls [4]. Others have reported a dysexecutive syndrome in the COVID patients [5]. Thus symptomatic patients do suffer cognitive deficits in addition to the earlier reported neurological features. Neurological features such as weakness, pain and numbness are reported readily as they are easily noticed by the patients. However dysfunctions of the higher mentation go unnoticed especially if they are mild and occur in otherwise asymptomatic persons [6]. Such unrecognised deficits have been brought out in asymptomatic subjects in many other diseases by targeted cognitive tests like MiniMental Status Examination (MMSE) and Montreal Cognitive Assessment(MoCA) [7, 8]. Although MMSE has been widely used for detecting cognitive impairment, it has been found inferior [9] to the MoCA in detecting Mild cognitive impairment (MCI). 
MoCA has been widely validated in diagnosing MCI in many studies [6]. We used the MoCA test to detect MCI in asymptomatic COVID-19 subjects.

As a case control study at the department of General Medicine, Great Eastern Medical School and Hospital, Ragole, India we compared the cognitive assessment scores of 93 asymptomatic COVID-19 subjects with those of 102 controls. The study was conducted from June 2020 to July 2020. Laboratory-confirmation of SARS-CoV-2 infection was done by RT-PCR on nasopharyngeal swabs. The subjects were recruited from relatives of COVID-19 patients brought to the hospital. Control subjects were negative for the infection. All subjects with age $<18$ years, comorbidities, psychiatric illness or if on any medication were excluded. The sample size was calculated to 91 cases and 91 controls based on the previous study by Zhou et al.We recruited 105 subjects in each group.The subjects were then administered the MoCA basic test in the local language as all of them had less than four years of education. MoCA includes nine domains: executive function, fluency, orientation, calculation, abstraction, delayed recall, visuoperception, naming and attention. Cognitive impairment is defined as score less than or equal to 26 out of 30.The scores were compared between cases and controls. Permission was obtained to use the MoCA for the study. The data was analyzed using IBM SPSS Statistics-version 21 (IBM Corp. Released 2012. IBM SPSS Statistics for Windows, Version 21.0. Armonk, NY: IBM Corp.). The data is presented as mean and standard deviation. Tests of significance used were t-test and one way ANOVA for parametric data. The data is available with the corresponding author. Written informed consent was taken from the subjects. The Institutional Ethical Committee at GEMS, Ragole approved the study. (89/IEC/GEMS\&H/2020).

In our study we finalised 93 asymptomatic COVID patients and 102 controls after exclusion due to lack of consent and insufficient data. The mean age of the patients was $36.2 \pm 11.7$ and that of the controls was $35.6 \pm 9.8$. In the CoViD subjects $52.3 \%$ were women and in the controls $54.7 \%$ were women.

There was no significant difference in the overall cognitive assessment scores between the two groups, but COVID-19 patients secured lower scores than controls in the domains of visuoperception, naming and fluency (Table 1).

Also, COVID positive subjects aged greater than 50 years scored lower in the MoCA when compared to the younger people. (Table 2). There were no differences between the two genders (Supplementary table).

\section{Cases}

\section{Tukey HSD post-hoc test...}

Age 18-29 versus Age 30-49: Diff $=0.0000,95 \% \mathrm{CI}=$ -1.3905 to $1.3905, \mathrm{p}=\mathrm{NaN}$.

Age 18-29 versus Age 50 and above: Diff $=-1.9000$, $95 \% \mathrm{CI}=-3.6515$ to $-0.1485, p=0.0302$.

Age 30-49 versus 50: Diff $=-1.9000,95 \% \mathrm{CI}=-$ 3.5840 to $-0.2160, p=0.0230$.

\section{Controls}

\section{Tukey HSD post-hoc test...}

Age 18-29 versuss Age 30-49: $\quad$ Diff $=-1.4000$, $95 \% \mathrm{CI}=-5.7355$ to $2.9355, p=0.6821$.

Age 18-29 versus Age 50 and above: Diff $=-2.5000$, $95 \% \mathrm{CI}=-9.1226$ to $4.1226, p=0.5961$.

Age 30-49 versus Age 50 and above: Diff $=-1.1000$, $95 \% \mathrm{CI}=-7.8166$ to $5.6166, p=0.9044$.

We found that asymptomatic COVID-19 patients had impairments in certain cognitive domains. They included visuoperception, naming and fluency. Older patients had more MCI compared to the subjects below 50 years of age.

Our study adds to the Zhou et al. study that showed defects of CPT in CoViD patients and extends the neurological spectrum even to otherwise asymptomatic subjects. These findings confirm the cognitive impairments due to COVID-19.

The dysfunction in multiple cognitive domains corresponds to dysfunction of multiple parts of the brain [10]. This is in line with previous neuropathological $[11,12]$ and neuroimaging studies [13] which have shown that viral pathology involves most of the brain. These neurological patterns have been associated with most of the corona viruses [14]. Direct neural invasion, neuroinflammation and systemic proinflammatory state and hypoxia have all been suggested to mediate the neurological dysfunction in COVID [15]. An interesting study has shown that asymptomatic subjects also may have pneumonia discernible only by imaging [16]. It may even be possible that the MCI in our COVID subjects might be due to impaired oxygen supply to the brain [15], keeping in mind that even hypoxia goes undetected in some patients even with a pulse oximeter [17].

Our finding of cognitive impairment in asymptomatic COVID patients is important especially in elderly patients if they already have MCI. It might be further compromised. Even without other symptoms they might thus need more 
Table 1 Montreal cognitive assessment scores of the subjects

\begin{tabular}{lcccc}
\hline Domain (Max. score) & $\begin{array}{c}\text { Asymptomatic COVID- } \\
\text { 19 subjects(93) }\end{array}$ & Controls(102) & $p$ value & 95\%CI \\
\hline Mean total score (30) & $26 \pm 2.6$ & $26.1 \pm 3.0$ & 0.887 & -1.492 to 1.292 \\
Executive function (1) & $0.9 \pm 0.2$ & $0.9 \pm 0.2$ & 1 & -0.105 to 0.105 \\
Fluency (2) & $0.9 \pm 0.6$ & $1.6 \pm 0.7$ & $<0.001$ & -1.002 to -0.378 \\
Orientation (6) & $5.6 \pm 0.6$ & $5.6 \pm 1$ & 1 & -0.353 to 0.353 \\
Calculation (3) & $2.4 \pm 0.8$ & $2.4 \pm 0.9$ & 1 & -0.426 to 0.426 \\
Abstraction (3) & $2.8 \pm 0.5$ & $2.6 \pm 1$ & 0.209 & -0.114 to 0.514 \\
Delayed recall (5) & $3.1 \pm 1.1$ & $3.2 \pm 1$ & 0.728 & -0.668 to 0.468 \\
Visuoperception (3) & $2.4 \pm 0.7$ & $2.8 \pm 0.7$ & 0.032 & -0.766 to -0.034 \\
Naming (4) & $3.6 \pm 0.5$ & $3.9 \pm 0.2$ & 0.016 & -0.545 to -0.055 \\
Attention (3) & $2.5 \pm 0.5$ & $2.6 \pm 0.8$ & 0.496 & -0.390 to 0.190 \\
\hline
\end{tabular}

Table 2 Age wise comparison of the MoCA scores

\begin{tabular}{lclrr}
\hline & Age 18-29 & $30-49$ & 50 and above & P value \\
\hline Mean MoCA Score in cases & $25.9 \pm 2.1$ & $25.9 \pm 2.3$ & $24 \pm 3.5$ & 0.018 \\
Mean MoCA score in controls & $27 \pm 1.7$ & $25.6 \pm 4.3$ & $24.5 \pm 3.5$ & 0.537 \\
\hline
\end{tabular}

Number of cases $=33$ (Age 18-29) versus 42(30-49) versus 18 (50 and above). Number of controls 48 versus 42 versus 12 vigilant care [18] as their activities of daily living(ADL) might be affected.

From our findings, it may be suggested that people employed in highly intellectual occupations might fall short of their work requirements if they have CoViD infection even asymptomatically. This is pertinent as doctors and medical personnel who are at a very high risk for the infection come under this category and their mental health condition is paramount to every medical strategy against CoVID 19.

Previous literature has hypothesized that COVID19 affected patients might be at high risk of developing cognitive decline after overcoming the primary infection [19]. The cognitive impairment reported in our study and the previous studies $[4,5]$ suggests the need for long term follow up of the COVID affected subjects as people with MCI are prone to dementia [20].

Our study is limited by the small sample and the hospital bias but it suggests MCI in asymptomatic COVID subjects. And since only one test was done, a full psychometric assessment study is warranted to fully understand the pattern better.

Acknowledgements We thank Dr Anikha Bellad MD for critical internal peer review.

Funding None.

Compliance with ethical standards
Conflicts of interest The authors declare that they have no conflict of interest.

\section{References}

1. Meier K, Glatz T, Guijt MC, Piccininni M, van der Meulen M, Atmar K, Jolink AT, Kurth T, Rohmann JL, Zamanipoor Najafabadi AH. COVID-19 Survey Study group. Public perspectives on protective measures during the COVID-19 pandemic in the Netherlands, Germany and Italy: A survey study. PLoS ONE. 2020;15(8):e0236917.

2. Asadi-Pooya AA, Simani L. Central nervous system manifestations of COVID-19: A systematic review. J Neurol Sci. 2020;11:116832.

3. Ahmad I, Rathore FA. Neurological manifestations and complications of COVID-19: A literature review. J Clin Neurosci. 2020.

4. Zhou H, Lu S, Chen J, Wei N, Wang D, Lyu H, Shi C, Hu S. The landscape of cognitive function in recovered COVID-19 patients. J Psychiatr Res. 2020.

5. Ardila A, Lahiri D. Executive dysfunction in COVID-19 patients. Diabetes Metab Syndr. 2020;14(5):1377-8.

6. Smith NA, Yeow YY. Use of the Montreal Cognitive Assessment test to investigate the prevalence of mild cognitive impairment in the elderly elective surgical population. Anaesth Intensive Care. 2016;44(5):581-6.

7. Martínez-Banfi M, Vélez JI, Perea MV, García R, Puentes-Rozo PJ, Mebarak Chams M, Ladera V. Neuropsychological performance in patients with asymptomatic HIV-1 infection. AIDS care. 2018;30(5):623-33.

8. Lal BK, Dux MC, Sikdar S, Goldstein C, Khan AA, Yokemick J, Zhao L. Asymptomatic carotid stenosis is associated with cognitive impairment. J Vasc Surg. 2017;66(4):1083-92. 
9. Bhat G, Yost G, Mahoney E. Cognitive function and left ventricular assist device implantation. J Heart Lung Transp. 2015;34(11):1398-405.

10. Julayanont P, Nasreddine ZS. Montreal Cognitive Assessment (MoCA): concept and clinical review. In Cognitive screening instruments. Springer: Cham; 2017. pp. 139-195

11. Reichard RR, Kashani KB, Boire NA, Constantopoulos E, Guo Y, Lucchinetti CF. Neuropathology of COVID-19: a spectrum of vascular and acute disseminated encephalomyelitis (ADEM)-like pathology. Acta Neuropathol. 2020;24:1-6.

12. Polak SB, Van Gool IC, Cohen D, Jan H, van Paassen J. A systematic review of pathological findings in COVID-19: a pathophysiological timeline and possible mechanisms of disease progression. Mod Pathol. 2020;22:1-1.

13. Kremer S, Lersy F, de Sèze J, Ferré JC, Maamar A, Carsin-Nicol B, Collange O, Bonneville F, Adam G, Martin-Blondel G, Rafiq M. Brain MRI findings in severe COVID-19: a retrospective observational study. Radiology. 2020;16:202222.

14. Katal S, Balakrishnan S, Gholamrezanezhad A. Neuroimaging findings in COVID-19 and other coronavirus infections: a systematic review in 116 patients. J Neuroradiol. 2020.

15. Yavarpour-Bali H, Ghasemi-Kasman M. Update on neurological manifestations of COVID-19. Life Sci. 2020;9:118063.
16. Hu Z, Song C, Xu C, Jin G, Chen Y, Xu X, Ma H, Chen W, Lin Y, Zheng Y, Wang J. Clinical characteristics of 24 asymptomatic infections with COVID-19 screened among close contacts in Nanjing. China Sci China Life Sci. 2020;63(5):706-11.

17. Ottestad W, Seim M, Mæhlen JO. COVID-19 with silent hypoxemia. Tidsskrift for Den norske legeforening. 2020.

18. Goodman-Casanova JM, Dura-Perez E, Guzman-Parra J, CuestaVargas A, Mayoral-Cleries F. Telehealth home support during COVID-19 confinement for community-dwelling older adults with mild cognitive impairment or mild dementia: survey study. J Med Int Res. 2020;22(5):e19434.

19. Heneka MT, Golenbock D, Latz E, Morgan D, Brown R. Immediate and long-term consequences of COVID-19 infections for the development of neurological disease. Alzheimer's Res Ther. 2020;12:1-3.

20. Saunders S, Ritchie K, Russ TC, Muniz-Terrera G, Ritchie CW. Evolution and future directions for the concept of mild cognitive impairment. Int Psychogeriatr. 2018;30(10):1431-4.

Publisher's Note Springer Nature remains neutral with regard to jurisdictional claims in published maps and institutional affiliations. 